\title{
功能碳基复合材料在锂硫电池正极中的应用
}

\author{
刘汉鼎 $a, b$ 贾国栋 $b \quad$ 朱胜 $b \quad$ 盛建 $b$ 张则尧 $a, b$ 李彦*, $, a, b$ \\ ( ${ }^{a}$ 北京大学深圳研究院 深圳 518057) \\ ( ${ }^{b}$ 北京分子科学国家研究中心 纳米器件物理与化学教育部重点实验室 稀土材料及应用国家重点实验室 \\ 北京大学化学与分子工程学院 北京 100871)
}

\begin{abstract}
摘要 碳基复合材料由于结构可变、形貌可调、成分可控, 能够展现出优异的理化特性, 在能源存储和转化领域具有 极大的应用潜力. 其中, 锂-硫电池作为高效的能源存储和转化器件, 长期受困于硫 $(\mathrm{S})$ 和硫化锂 $\left(\mathrm{Li}_{2} \mathrm{~S}\right)$ 绝缘的瓶颈, 亟 需开发高导电的储硫载体帮助锂-硫电池实现可逆充放电. 研究表明, 碳基复合材料具有强的导电能力, 且可以通过 表/界面和缺陷工程的技术制备获得, 易于实现多功能的耦合, 能够显著改善长循环中硫正极的流失, 缓解容量和倍率 的衰减. 本综述通过总结碳基复合材料在锂-硫电池中的应用研究进展, 详细讨论功能化的碳基储硫载体和硫正极电 化学性能的构-效关系, 分析材料的形貌、结构和组分的改变对硫正极电化学性能的影响. 最后, 对功能化碳基复合材 料在锂-硫电池的实用化发展方向进行了展望.
\end{abstract}

关键词 碳基复合材料; 锂-硫电池; 功能化; 正极

\section{Functionalized Carbon-Based Composite Materials for Cathode Application of Lithium-Sulfur Batteries}

\author{
Liu, Handing ${ }^{a, b} \quad$ Jia, Guodong $^{b} \quad$ Zhu, Sheng $^{b} \quad$ Sheng, Jian $^{b} \quad$ Zhang, Zeyao ${ }^{a, b} \quad$ Li, Yan*,a,b \\ ( ${ }^{a}$ Peking University Shenzhen Institute, Shenzhen 518057, China) \\ ( ${ }^{b}$ Beijing National Laboratory for Molecular Science, Key Laboratory for the Physics and Chemistry of Nanodevices, \\ State Key Laboratory of Rare Earth Materials Chemistry and Applications, College of Chemistry and Molecular Engineering, \\ Peking University, Beijing 100871, China)
}

\begin{abstract}
Carbon-based materials exhibit excellent physical and chemical properties due to their diverse structure, adjustable morphology and controllable composition. They have great application potentials in the field of new energy storage and conversion. Lithium-sulfur batteries as a kind of important new energy storage devices are trapped by the sulfur and lithium sulfide bottleneck of electronic insulation, which leads to irreversible charging and discharging processes. Fortunately, carbon-based composite materials have strong electrical conductivity. The coupling of multi-functions of the composite materials can improve the long-term utilization of sulfur and alleviate the attenuation of cycle and rate capacity. Functionalized carbon-based composite materials can be prepared through surface/interface and defect engineering techniques, which have attracted the attention and favor of scientific researchers. We review the preparation methods of functionalized carbon-based host materials and analyze the structure-function relationship of carbon-based composite materials and sulfur cathodes. We discuss the influence of the change of morphology, structure, and composition on the electrochemical performance of sulfur cathode. Finally, the feasibility of the functionalized carbon-based composite materials are prospected in the practical application of the lithium-sulfur battery.
\end{abstract}

Keywords carbon-based composite material; lithium-sulfur battery; functional; cathode

\section{1 引言}

开发持续稳定、绿色环保的新型清洁能源替代传统 化石能源是实现人类社会可持续发展的关键途径 ${ }^{[1]}$. 目
前, 人们开发的新型能源受到自然环境、生态干扰和时 空差异的不利影响, 而无法高效、安全地进行能量转化, 严重制约了新型清洁能源规模化应用 ${ }^{[2]}$. 为了克服上述 困难, 将新型清洁能源转化为电能进行存储和消纳是可

*E-mail: yanli@pku.edu.cn; Tel.: 010-62756773

Received August 14, 2021; published November 12, 2021.

Project supported by the Shenzhen Basic Research Project (No. JCYJ20170817113121505), the Shenzhen KQTD Project (No. KQTD20180411143400981), the National Science and Technology Major Project of the Ministry of Science and Technology of China (No. 2016YFA0201904), the National Natural Science Foundation of China (Nos. 21631002, 52101209), the Beijing National Laboratory for Molecular Sciences (No. BNLMS-CXTD-202001), and the China Postdoctoral Science Foundation (No. 2020M682606).

深圳市科技计划项目(No. JCYJ20170817113121505)、深圳市海外高层次人才创新创业专项资金(No. KQTD20180411143400981)、科技部国家科技重 大专项(No. 2016YFA0201904)、国家自然科学基金(Nos. 21631002, 52101209)、北京分子科学国家研究中心(No. BNLMS-CXTD-202001)和中国博士 后面上基金项目(No. 2020M682606)资助. 
行策略. 因此, 高比能是储能器件应用的重要前提. 锂硫电池 $(\mathrm{Li}-\mathrm{S})$ 的正负极材料同时具有高比能的天然优势, 在新型清洁能源的转化中被科研和产业界同时寄于厚 望 ${ }^{[3]}$.

一方面, 在地壳中硫的储量丰富易于获得; 另一方 面, 硫单质兼具高理论比容量 $\left(1675 \mathrm{mAh} \cdot \mathrm{g}^{-1}\right)$ 和能量密 度 $\left(2600 \mathrm{Wh} \cdot \mathrm{kg}^{-1}\right)$ 的双重优势, 可以在大型供能设备的 储能段提供稳定持续的能量供给 ${ }^{[4-5]}$. 遗憾的是, 硫本征 导电性差, 电子迁移能力弱, 无法实现锂-硫电池的可 逆充放电循环. 利用高导电的碳基材料作为储硫载体, 构建碳-硫复合正极能够大幅提升电池的可逆循环性能, 但是在充放电过程中相变反应复杂, 而且中间态的各相 行为多变. 其中初始 $\mathrm{S}_{8}$ 和终态的 $\mathrm{Li}_{2} \mathrm{~S}$ 及其中间态 $\mathrm{Li}_{2} \mathrm{~S}_{2}$ 均为电子绝缘体, 易团聚钝化, 溶液相中间态的多硫化 锂易穿梭溶解, 均会造成活性物质硫单质的不可逆损 失 ${ }^{[6]}$. 因此, 开发先进的碳-硫复合体系来提升锂-硫电 池的电化学性能成为了关键.

碳基复合载体，通常由碳基材料和各种金属基材料 复合而成. 使得复合材料组分整体可变, 形貌均匀可 控、结构灵活可调, 不仅能够整合多维分级结构的维度 优势, 而且能够调制不同组分的理化特性, 协同实现复 合材料的功能叠加, 从而进一步提升电子的高效输运, 以及对溶液相多硫化锂进行有效的功能调制, 促进硫正 极的高效转化 ${ }^{[7]}$. 基于此, 本文作者所在课题组从硫正 极实用化的视角出发, 综述了多功能碳基复合材料在 锂-硫电池中的应用进展, 综合考虑碳基复合材料的形 貌学(多维分级设计)、几何结构(孔隙率、比表面积)和 组分调制(掺杂修饰与功能的构-效关系)对锂硫电池硫 正极实用化性能(高硫含量、厚面积担载量和低电解液 用量/硫正极载量 $\mathrm{E} / \mathrm{S}$ 比)的整体影响. 提出轻质/多功能 化的碳基复合材料是加快锂硫电池硫正极性能实用化 进程的可行策略, 并对该类碳基储硫材料在锂-硫电池 中的应用前景做了进一步展望.

\section{2 锂-硫电池的反应机制表征及挑战}

锂-硫电池在充电过程中对应于硫正极脱锂化反应, 放电过程对应嵌锂反应, 总的反应方程式可以表达为: $\mathrm{S}_{8}+16 \mathrm{Li}^{+}+16 \mathrm{e}^{-} \leftrightarrow 8 \mathrm{Li}_{2} \mathrm{~S}^{[8-17]}$. 充电过程中, $\mathrm{Li}_{2} \mathrm{~S}$ 脱锂为 单质硫. 放电过程中反应复杂, 由多种固相和液相的相 转变构成, 涉及初始/中间/终态六种产物的衍变 ${ }^{[18-20]}$ :

(1)固-液相转变: $\mathrm{S}_{8} \rightarrow \mathrm{Li}_{2} \mathrm{~S}_{8}$.

(2)液-液相转变: $\mathrm{Li}_{2} \mathrm{~S}_{8} \rightarrow \mathrm{Li}_{2} \mathrm{~S}_{x}(4 \leqslant x \leqslant 8)$.

(3)液-固相转变: $\mathrm{Li}_{2} \mathrm{~S}_{x}(4 \leqslant x \leqslant 8) \rightarrow \mathrm{Li}_{2} \mathrm{~S}_{2}$.

(4)固-固相转变: $\mathrm{Li}_{2} \mathrm{~S}_{2} \rightarrow \mathrm{Li}_{2} \mathrm{~S}$

在负极侧的固-液界面处, 金属锂负极不稳定. 在 充电中金属锂表面发生不均匀的沉积聚集成树突形状 的枝晶, 形成了一层 SEI (solid electrolyte interface)膜. 这些 SEI 膜的亲锂特性会吸纳更多的锂沉积并诱导 SEI
膜破裂, 会刺穿隔膜引起电池发生短路, 给电池的持续 运行增加安全隐患 ${ }^{[21-22]}$. 醚类电解液不仅要负责锂离子 的高效运输, 而且还要和溶液相中间态的多硫化锂互相 兼容 ${ }^{[23-24]}$. 因此，需要调控商用醚类电解液的黏度和极 性，从而提升锂-硫电池循环实用化性能 ${ }^{[25-26]}$. 同样，在 正负极之间的隔膜阻断电池两极的直接接触, 防止电池 的内部短路, 并要让电解液中的锂离子顺利通过, 保证 电池内部回路的畅通.

\section{1 锂-硫电池反应机制的表征技术}

经过多年的研究, 科研工作者对理-硫电池工作原 理的了解逐渐深入，发现基于原位表征技术可以对理硫 电池体系中电化学过程进行精准的解析. 例如, 原位拉 曼光谱可以收集极化的正极表面和液体有机电解液中 时间和空间分辨的实时信息, 从而准确判断电位驱动下 硫正极及其中间相的生成动力学 ${ }^{[27]}$. 多硫化锂中 $\mathrm{S}^{2-}$ 阴 离子和自由基的拉曼谱峰均在 $550 \mathrm{~cm}^{-1}$ 波数以下, 其中 $\mathrm{S}_{8}$ 的特征峰在 $150 、 219$ 和 $474 \mathrm{~cm}^{-1}$; 高阶多硫化锂中的 阴离子 $\left(\mathrm{S}_{8}{ }^{2-} 、 \mathrm{~S}_{6}{ }^{2-}\right.$ 和 $\left.\mathrm{S}_{4}{ }^{2-}\right)$ 也可以利用拉曼光谱进行检测; 此外, $\mathrm{S}_{3}$ 自由基在 $525 \sim 535 \mathrm{~cm}^{-1}$ 内具有特征信号 ${ }^{[28]}$. 原 位红外光谱技术能够区分不同多硫化锂物种中 $S-S$ 键 的振动模式. 多硫化锂 $\left(\mathrm{Li}_{2} \mathrm{~S}_{x}\right)$, 其中 $x=2 \sim 8$ 的特征峰 依次为: $\mathrm{Li}_{2} \mathrm{~S}_{2}$ 约 $475.8 \mathrm{~cm}^{-1}, \mathrm{Li}_{2} \mathrm{~S}_{3}$ 约 $479.5 \mathrm{~cm}^{-1}, \mathrm{Li}_{2} \mathrm{~S}_{3.5}$ ( $^{*}$ 表示为两种多硫化物的混合相)约 $483.3 \mathrm{~cm}^{-1}, \mathrm{Li}_{2} \mathrm{~S}_{4.5^{*}}$ 约 $487.8 \mathrm{~cm}^{-1}, \mathrm{Li}_{2} \mathrm{~S}_{5}$ 约 $490.6 \mathrm{~cm}^{-1} 、 \mathrm{Li}_{2} \mathrm{~S}_{6}$ 约 $495.2 \mathrm{~cm}^{-1}$ 和 $\mathrm{Li}_{2} \mathrm{~S}_{8}$ 约 $504.0 \mathrm{~cm}^{-1}$ 范围 ${ }^{[29-30]}$. 此外, 红外光谱还能对电 解液中三氟甲磺酸盐阴离子配位状态进行清晰地监 测 ${ }^{[29]}$. 在原位紫外光谱中位于 $70 \sim 280 \mathrm{~nm}$ 范围内弱的 特征吸收峰表明 $\mathrm{S}_{8}$ 在电解液中有极低的溶解度. 而中 间相的多硫化锂中的 $\mathrm{S}^{2-}$ 阴离子在 350 到 $500 \mathrm{~nm}$ 范围内 有很强的吸收峰, 中间态 $\mathrm{S}_{3}$ 自由基在 $620 \mathrm{~nm}$ 处有强吸 收 ${ }^{[31]}$. 而且长链的多硫化锂往往在长波长处具有吸收 峰. 例如, $\mathrm{Li}_{2} \mathrm{~S}_{8}$ 的吸收峰位于约 $500 \mathrm{~nm}$, 而 $\mathrm{Li}_{2} \mathrm{~S}_{4}$ 的特征 峰位于约 $400 \mathrm{~nm}$ 附近 ${ }^{[32]}$.

\section{2 锂硫电池正极材料的挑战}

硫正极由于本征电子传输能力差, 电导率仅为 $10^{-30} \mathrm{~S} \cdot \mathrm{m}^{-1}$, 无法满足二次可逆充放电的需求. 碳基复 合材料普遍具有较高的电子迁移能力, 且亲硫性强, 为 解决硫正极无法可逆充放电的循环提供了可行途径. 然 而，碳-硫正极在长循环中依然存在以下三个方面的问 题: (1)完全锂化后所生成的终态产物硫化锂 $\left(\mathrm{Li}_{2} \mathrm{~S}\right)$, 体 积膨胀率达到 $80 \%$, 严重影响电极材料的结构完整性. (2)随着电解液中溶液相中间态的多硫化锂的溶解，导 致浓差极化加重, 终态电子绝缘的硫化锂表面容易致密 化, 进而形成一层厚厚的钝化层, 均会降低转化动力学 速率，严重削弱了硫单质的有效利用率. (3)硫正极放电 过程中的中间产物极易溶解在商用醚类电解液中, 随着 电解液的流动穿梭至负极侧腐蚀锂, 会引起严重的性能 
衰减和安全隐患 ${ }^{[33]}$. 如何从形貌构建、结构设计和成分 优化三个层面出发, 构建轻质/多功能位点的碳基复合 材料是提升锂-硫电池实用化性能(高硫含量、厚面积担 载量和低电解液用量/硫正极含量 $\mathrm{E} / \mathrm{S}$ 比)的必由之路.

\section{3 功能碳基复合材料储硫正极的研究进展}

碳基复合材料具有组分丰富、形貌多样、结构稳定 以及易于修饰改性等特点, 被广泛用于硫正极载体材 料 ${ }^{[34-42]}$. 根据高性能储硫载体所需要的高导电、强固硫 和快催化的三功能要求(图 1), 本课题组将从碳基复合 材料的形貌构建、结构设计以及组分优化等方面分别论 述多功能的复合碳基载体材料在锂-硫电池中的研究进 展.

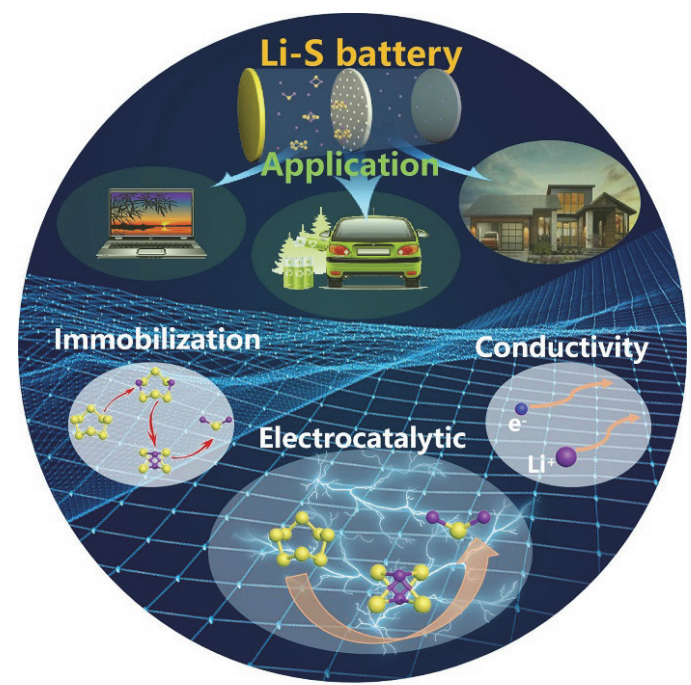

图 1 先进碳基复合储硫载体的三功能化设计与应用

Figure 1 Tri-functional design and application of an advanced carbonbased composite sulfur storage host

\section{1 单功能导电碳基储硫正极的研究进展}

提升碳基材料的石墨化程度，优化材料的空间形貌 (如形成三维交联互通的空间网络), 构建杂原子掺杂 $(\mathrm{N} / \mathrm{P} / \mathrm{S})$ 均能够有助于电子的快速定向输运, 可以显著提
升碳基材料的储硫性能 ${ }^{[41,43-48]}$. 2009 年 Nazar 课题组 ${ }^{[6]}$ 设计出一种高度有序的介孔碳管, 通过浸渍将硫熔融渗 透进入一维介孔碳管(CMK-3)中, 利用高度有序的介 孔碳与硫正极的充分浸润，实现了一维碳基材料在锂硫电池中的可逆循环，100 个周期后依然具有 390 $\mathrm{mAh} \cdot \mathrm{g}^{-1}$ 的放电容量. 随后, Zhang 等 ${ }^{[47]}$ 将碳纳米管设 计为 “薯条” 形状，中空的碳管内部可用于存储单质 硫，外表的介孔可促进锂离子的迁移(如图 2 所示). 与 只有单一介孔的 CMK-3 相比, 中空的大孔不仅有利于 获得高硫载量, 而且可预留空间缓解硫化锂的体积膨 胀效应。该复合硫正极经过 180 个周期循环后，容量 依然具有 $460 \mathrm{mAh} \cdot \mathrm{g}^{-1}$, 每圈的容量衰减率为 $0.3 \%$. 相 比单一介孔碳-硫正极, 该复合正极具有更稳定的循环 能力. 为了进一步提升锂硫电池的首圈容量低的问题, Zhi 和 $\mathrm{Xiao}^{[48]}$ 首先设计出具有 “胶囊” 形貌的硫正极 载体, 通过精确调控胶囊壁的直径和孔的分布, 增大 硫与载体的接触表面积, 并利用高的孔隙率实现载体 的高硫负载(质量分数 $76 \%$ ). 基于碳基材料几何结构 的优化, 该复合正极在 $0.5 \mathrm{C}$ 的电流密度下将首圈放电 容量提升至 $1165 \mathrm{mAh} \cdot \mathrm{g}^{-1}$. 考虑到长循环中多硫化锂在 电解液中的穿梭会引起电池容量的快速衰减, $\mathrm{Xu}$ 和 Guan 等[49]利用模板牺牲策略制备出具有核壳结构的碳 基纳米材料，除了分级多孔和表面结构优势，核壳结构 的 “空间限域” 效应能够有效约束多硫化锂在电解液中 的溶解扩散. 复合电极循环 200 个周期后依然具有 647 $\mathrm{mAh} \cdot \mathrm{g}^{-1}$ 的可逆容量, 具有更加稳定的循环稳定性能和 高的库伦效率.

虽然碳基材料的形貌工程能够显著提升碳-硫正极 的循环性能, 但是表面缺少功能位点的石墨化碳基载体 很难满足实用化条件(如高硫载量)下的循环倍率性 能 ${ }^{50]}$. 其中, 通过对表面的碳原子进行杂原子修饰, 形 成缺陷构筑功能位点, 既能诱导硫单质均匀地分布在载 体表面，又能加强碳基体对硫及中间相的化学吸附作用 (如图 3), 提升长周期循环中硫正极的利用效率 ${ }^{[3,51-54]}$. 窦世学课题组 ${ }^{[55]}$ 在石墨化碳的表面引入具有吸电子的

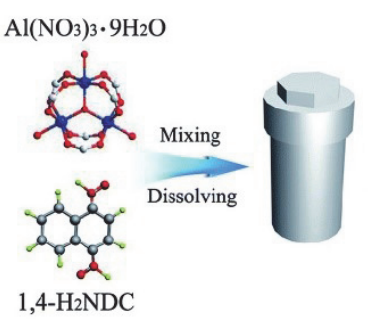

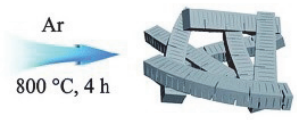

FLHPC
Hydrothermal

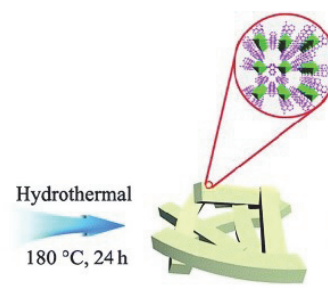

Al-MOF
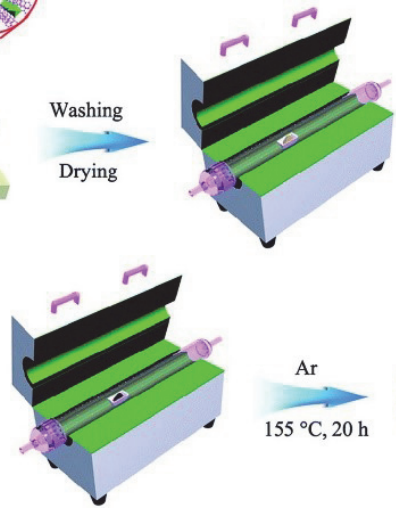

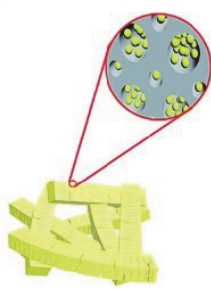

S/FLHPC

图 $2 \mathrm{~S} / \mathrm{FLHPC}$ 复合材料制备示意图 [47]

Figure 2 Schematic illustration of the S/FLHPC composite preparation ${ }^{[47]}$ 

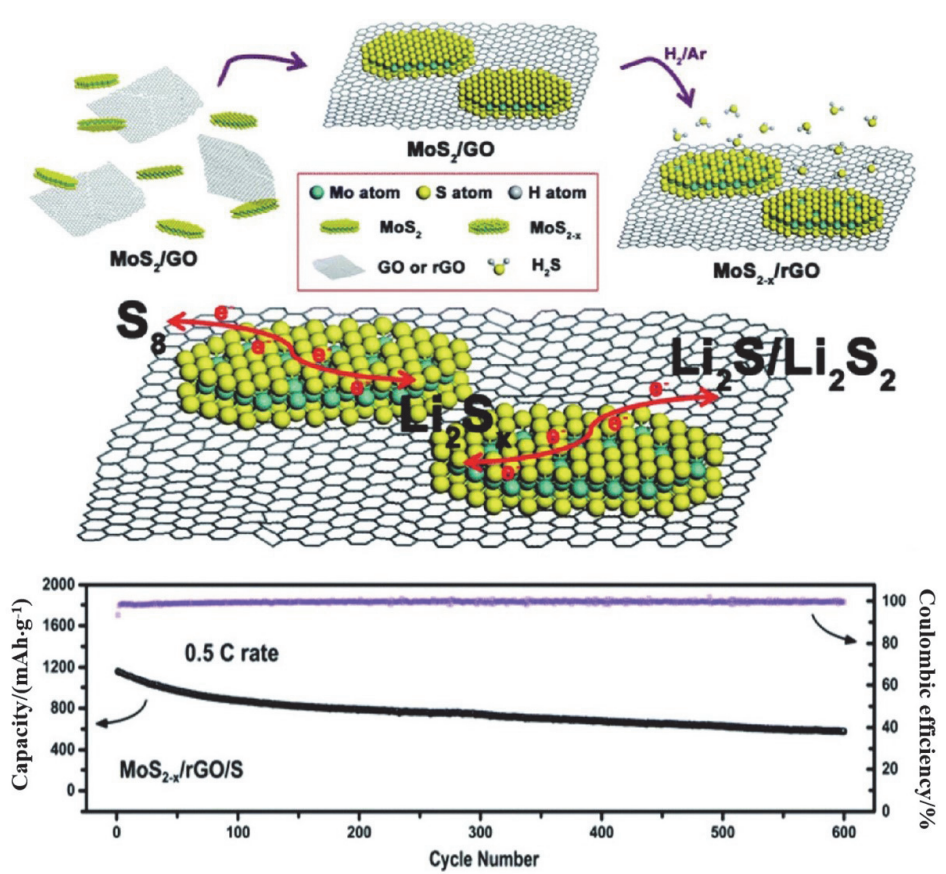

图 3 二维碳基复合载体对硫正极功能化约束示意图 ${ }^{[54]}$

Figure 3 Constraint schematic diagram of two-dimensional carbon-based composite host on the functionalization for sulfur cathode ${ }^{[54]}$

官能基团, 当载体中硫含量达到质量分数 $93 \%$, 该复合 正极在 $0.1 \mathrm{C}$ 的电流密度下可以稳定循环 200 个周期, 可逆容量的留存率达到 $731 \mathrm{mAh} \cdot \mathrm{g}^{-1}$, 平均库仑效率高 达 $99.2 \%$, 平均容量衰减率为 $0.013 \%$ (图 4).

一方面，设计出具有丰富的分级孔结构的碳基材 料，能够从几何空间上调制载体的孔隙率和孔体积，优 化碳基复合材料的储硫量和载流子的迁移速率 ${ }^{[56]}$. 另 一方面, 在碳基材料表面可掺杂各类杂原子, 形成具有
缺陷的碳结构, 这些杂原子掺杂的碳基材料能够与溶液 相中间态的多硫化锂形成更强的化学吸附，可进一步提 高硫正极的循环稳定性 ${ }^{[56]}$. Wang 等 ${ }^{[56]}$ 对介孔碳进行了 氮/氧原子的共掺修饰，通过直观的吸附实验和第一性 原理计算结果同时表明碳基材料中负电性的杂原子对 于溶液相中间态多硫化锂产生较强的化学吸附作用，可 以更加强有力地吸附多硫化锂, 能够约束多硫化锂的溶 解和穿梭. 当电极材料的面积担载量达到 $>5 \mathrm{mg} \cdot \mathrm{cm}^{-2}$

(a)

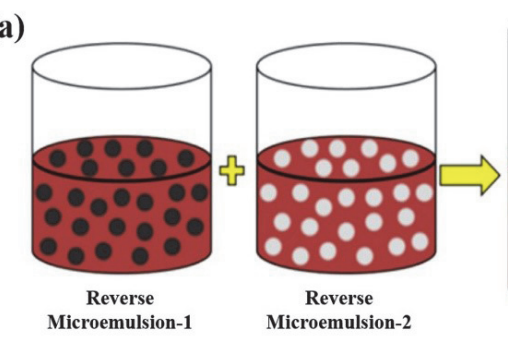

(b)
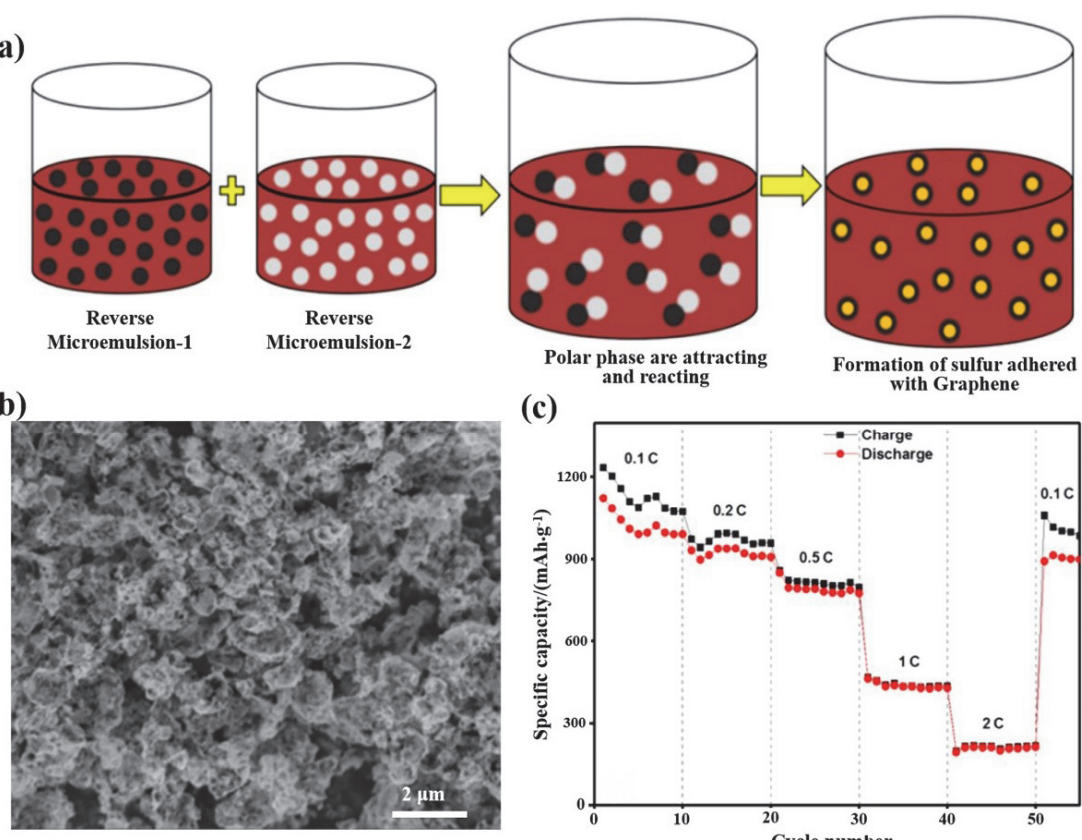

(c)

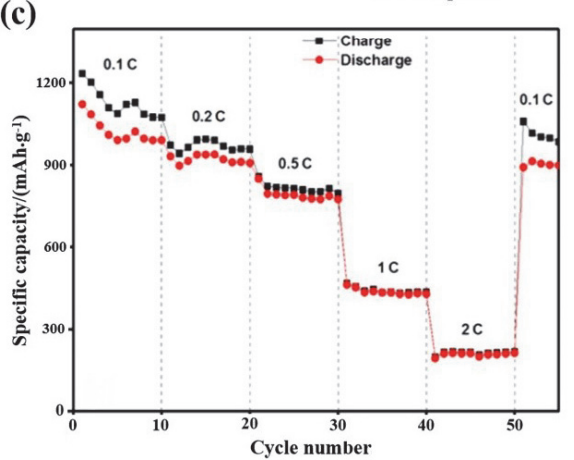

图 4 反相微乳液方法示意图: (a) 制备微乳液-1, (b) 制备微乳液-2, (c) 硫/石墨烯复合材料的合成 ${ }^{[55]}$

Figure 4 Schematic illustration of the reverse microemulsion method: (a) preparing microemulsion-1, (b) preparing microemulsion-2, and (c) synthesis of sulfur/graphene composite ${ }^{[55]}$ 
时, 放电比容量提升至 $1200 \mathrm{mAh} \cdot \mathrm{g}^{-1}$.

\section{2 多功能导电碳基储硫正极的研究进展}

通过对高导电的石墨碳基载体进行固硫的功能化 修饰, 能够提升长循环中电池的容量和利用效率, 但是 在电池长周期运行中, 随着充放电的深度进行, 电化学 反应的浓差极化不断增加, 反应动力学逐渐变慢, 钝化 现象加重 ${ }^{[57-58]}$. 开发能够催化电化学反应动力学速率的 载体至关重要. 此外, 锂硫电池的电极在高面积载量 $\left(>2 \mathrm{mg} \cdot \mathrm{cm}^{-2}\right)$ 下依然具有高能量密度 ${ }^{[59]}$. 因此, 在石墨 碳基材料上耦合多功能(固硫/催化)的金属化合物是提 升锂硫电池性能的有效策略 ${ }^{[60-67]}$. 楼雄文课题组 ${ }^{[58]}$ 将单 斜晶系的 $\mathrm{MnO}_{2}$ 纳米片填充到中空的石墨化碳纳米纤维 中 $\left(\mathrm{MnO}_{2} @ \mathrm{HCF}\right)$ 作为硫正极的载体材料(图 5). 三维交 联的导电网络, 有利于电子和离子的长距离迁移. 填充 的二氧化锰可将易溶相的多硫化锂约束在空心碳纤维 的内部. 而且, 每个中空碳纳米纤维都充当独立的纳米 级反应腔. 当复合电极中负载高面积硫载量时, 能够释 放出 $1161 \mathrm{mAh} \cdot \mathrm{g}^{-1}$, 相当于面积容量为 $3.8 \mathrm{mAh} \cdot \mathrm{cm}^{-2}$, 在 $0.5 \mathrm{C}$ 的大电流密度下稳定循环 300 个周期后依然具 有 $662 \mathrm{mAh} \cdot \mathrm{g}^{-1}$ 的放电容量.

Manthiram 课题组 ${ }^{[61]}$ 选择高极性的 $\mathrm{Fe}_{3} \mathrm{O}_{4}$ 作为功能 位点吸附多硫化锂, 将硫单质限域在具有三维多孔蛋黄 壳结构的纳米盒内部 YSC@ $\mathrm{Fe}_{3} \mathrm{O}_{4}$. 通过极性 $\mathrm{Fe}_{3} \mathrm{O}_{4}$ 与多 硫化锂的强化学相互作用, 将固定多硫化锂的效果最大 化. 同时, $\mathrm{Fe}_{3} \mathrm{O}_{4}$ 能够加快液相多硫化锂 $\mathrm{Li}_{2} \mathrm{~S}_{6}$ 的转化(图 $6)$. 当单位面积的硫载量为 $2.2 \mathrm{mg} \bullet \mathrm{cm}^{-2}$ 具有 1366
$\mathrm{mAh} \cdot \mathrm{g}^{-1}$ 的初始放电容量，同时经过 200 次循环后，容 量留存率为 $85.3 \%$, 平均每周期的容量衰减率仅为 $0.07 \%$.

\section{3 轻质/多功能导电碳基储硫正极的研究进展}

虽然极性金属化合物原位耦合碳基材料可以提升 硫正极的导电能力, 增强导电碳基载体的固硫/催化功 能. 但是, 在硫正极复杂的相变和多步的转化中, 需要 较长的驰豫时间, 长循环中硫正极仍会流失 ${ }^{[65-70]}$. 金属 化合物有限的催化功能只能减缓电池的容量衰减，并不 能实现长循环 ( $>500$ 周期以上)的稳定性 ${ }^{[10,71-74]}$. 而且石 墨化碳基材料孔隙率高, 在装配电池工艺中所需电解液 用量高, 即低电解液用量/硫正极质量 $\mathrm{E} / \mathrm{S}$ 比高, 会降低 液态电池的安全性 ${ }^{[59]}$. 因此, 实现载体的催化功能最大 化和增强碳基载体的振实密度是提升硫正极利用率、实 现更长周期下的稳定循环和倍率性能的关键 ${ }^{[74-76]}$. 基于 此, 质量占比低/催化功能强的载体设计尤为重要 ${ }^{[77-79]}$.

$\mathrm{Du}$ 等 ${ }^{[80]}$ 制备出大量 $\mathrm{Co}$ 单原子嵌入氮掺杂石墨烯 骨架内 $(\mathrm{Co}-\mathrm{N} / \mathrm{G})$, 通过透射电镜(TEM) 和 X 射线吸收光 谱法(XAS) 分析确定 Co 单原子嵌入 $\mathrm{N}$ 掺杂石墨烯的晶 格中, 并且与周围的 $\mathrm{N}$ 原子配位形成 Co-N-C 配位中心, 两个相邻配位中心 $\mathrm{Co}$ 原子之间的平均距离约为 $1 \mathrm{~nm}$. 结合电化学数据和第一性原理计算表明 Co-N-C 作为催 化活性位点, 在放电过程中促进固相 $\mathrm{Li}_{2} \mathrm{~S}$ 的形成, 同时 在充电过程也加速了固相 $\mathrm{Li}_{2} \mathrm{~S}$ 分解(图 7). 基于此, 利 用 $\mathrm{Co}-\mathrm{N} / \mathrm{G}$ 作为硫正极的载体，获得了 $90 \%$ 的超高载硫 量. 当电极片中硫的面积担载量为 $2.0 \mathrm{mg} \cdot \mathrm{cm}^{-2}$ 时, 在电

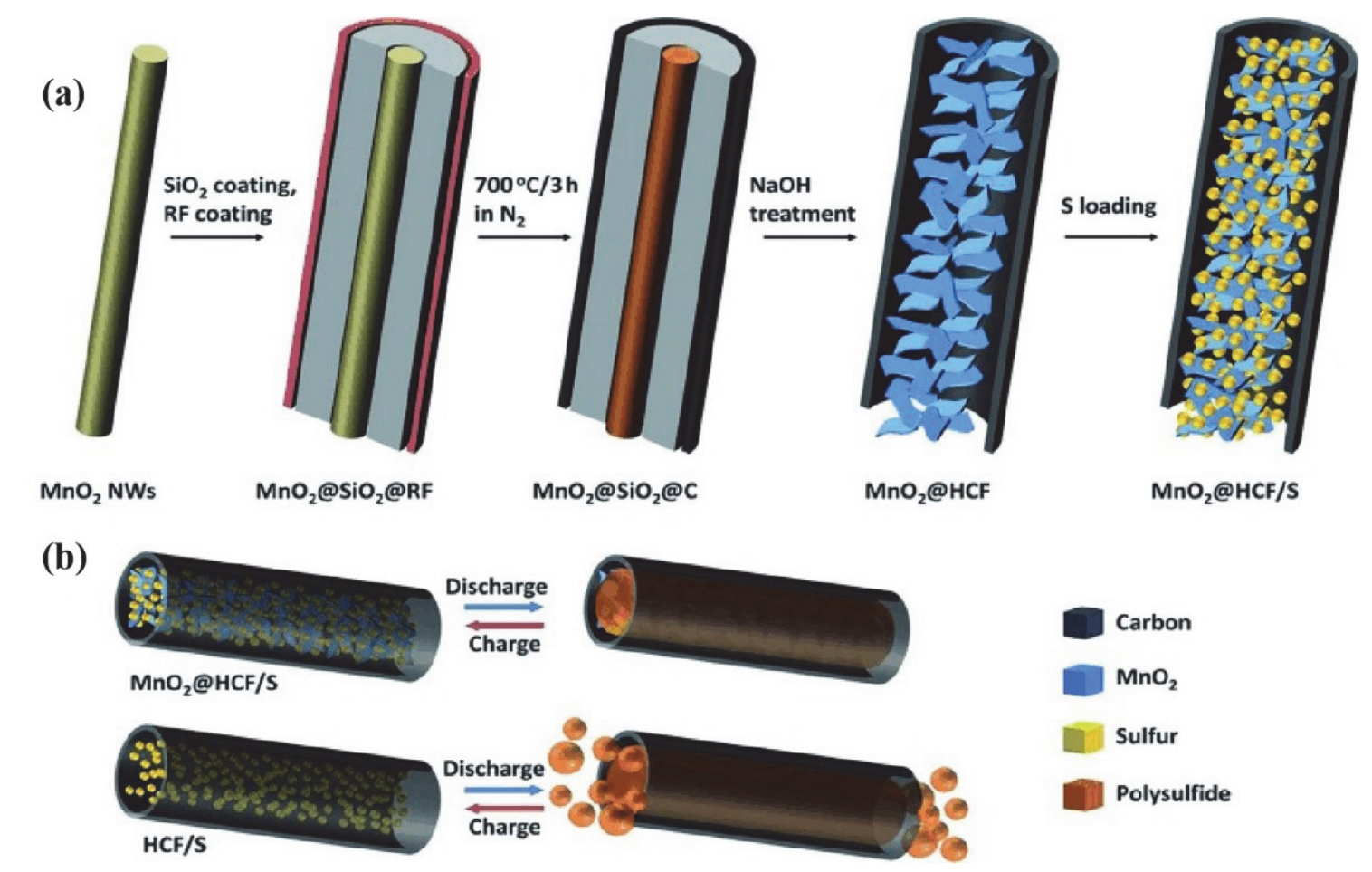

图 5 (a) $\mathrm{MnO}_{2} @ \mathrm{HCF} / \mathrm{S}$ 电极的合成示意图, (b)与 $\mathrm{HCF} / \mathrm{S}$ 正极相比的优势 $[58]$

Figure 5 (a) Synthesis of the $\mathrm{MnO}_{2} @ \mathrm{HCF} / \mathrm{S}$ composite, (b) advantages of the $\mathrm{MnO}_{2} @ \mathrm{HCF} / \mathrm{S}$ composite over $\mathrm{HCF} / \mathrm{S}^{[58]}$ 
(a)

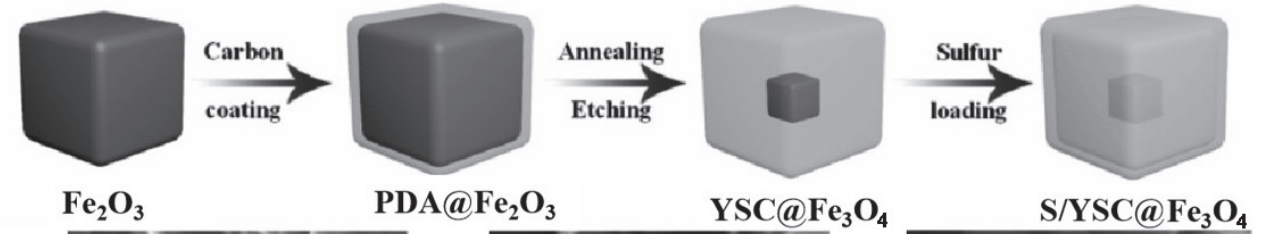

(b)
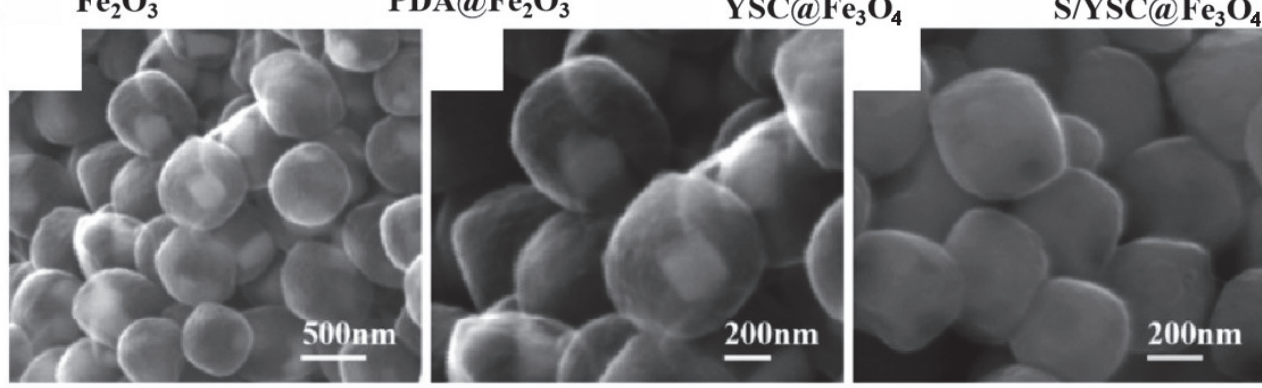

(c)

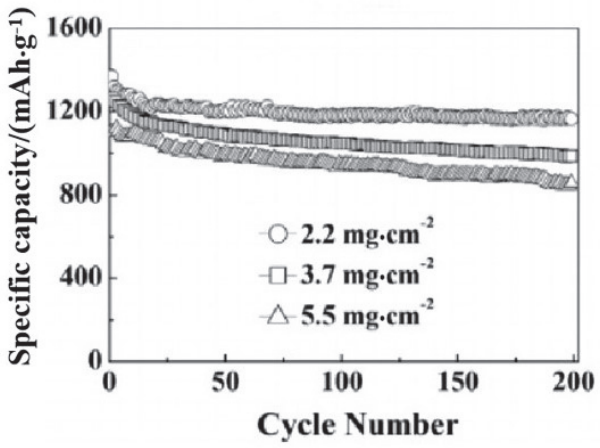

(d)

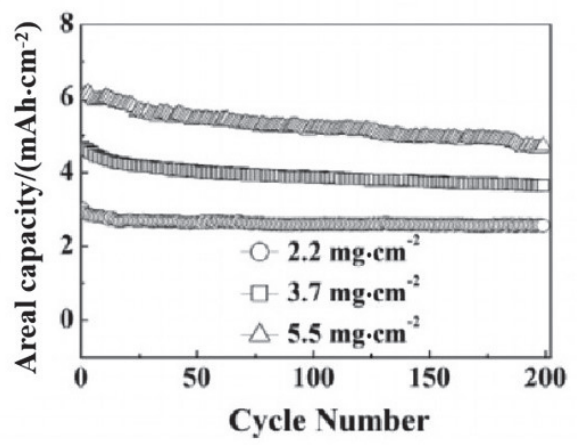

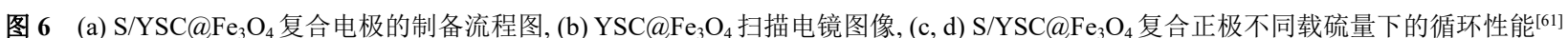

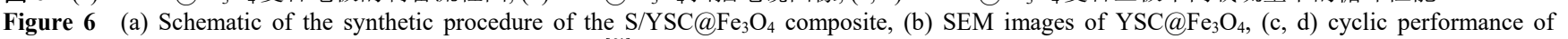
$\mathrm{S} / \mathrm{YSC} @ \mathrm{Fe}_{3} \mathrm{O}_{4}$ composite cathode with different sulfur load ${ }^{[61]}$

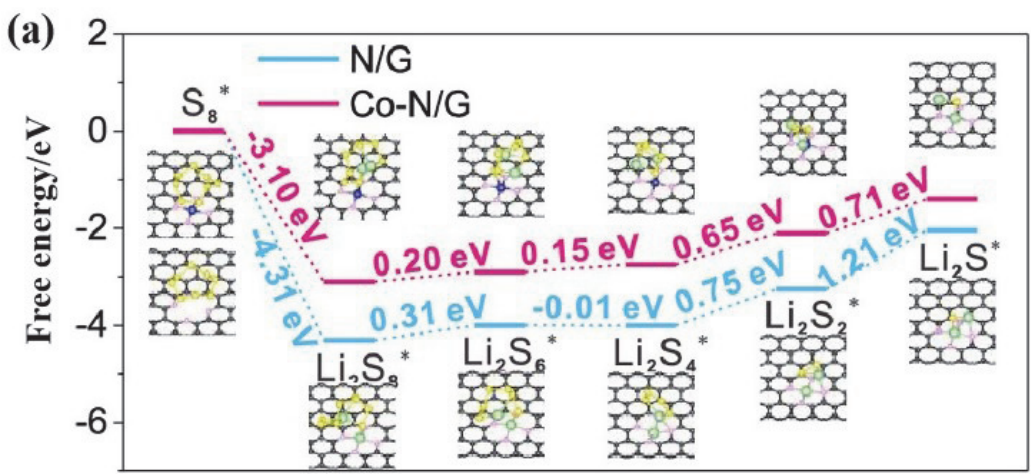

Reaction coordinate

(b)

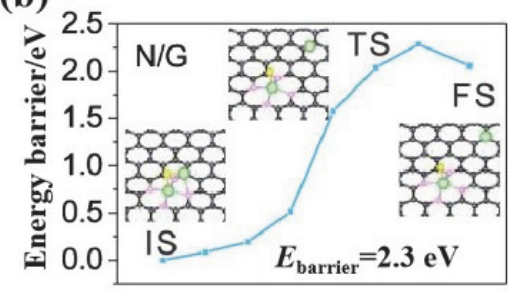

Reaction coordinate (c)

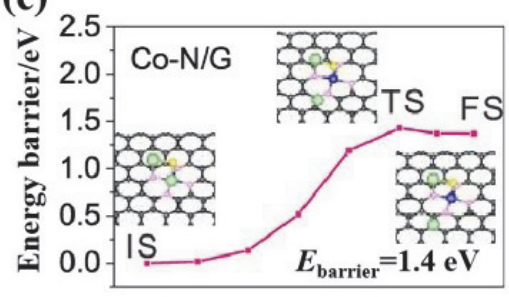

Reaction coordinate (d)
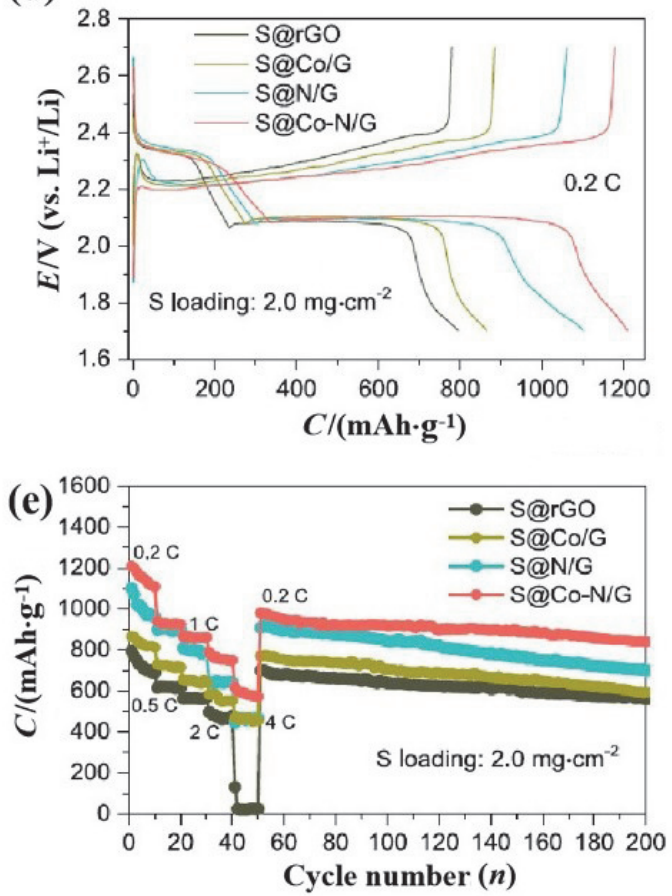

图 7 (a)在 $\mathrm{N} / \mathrm{G}$ 和 $\mathrm{Co}-\mathrm{N} / \mathrm{G}$ 基底上的还原中间相多硫化锂的能量分布图, (b, c) 在 N/G 和 Co- $\mathrm{N} / \mathrm{G}$ 基底上的固态 $\mathrm{Li}_{2} \mathrm{~S}$ 分解的能量分布图, $\mathrm{S} @ \mathrm{Co}-\mathrm{N} / \mathrm{G}$ 正极的电化学性能: (d)恒电流放电-充电曲线, (e)倍率性能 ${ }^{800}$

Figure 7 (a) Energy profiles for the reduction of LiPS on N/G and Co-N/G substrates, (b, c) energy profiles of the decomposition of $\mathrm{Li}_{2} \mathrm{~S}$ cluster on N/G and Co-N/G, electrochemical performance of S@Co-N/G cathode: (d) discharge-charge profiles, (e) rate capabilities ${ }^{[80]}$ 
流密度为 $0.2 \mathrm{C}$ 下释放出 $1210 \mathrm{mAh} \cdot \mathrm{g}^{-1}$ 比容量, 经过 100 个周期的稳定循环, 平均每圈的容量衰减率为 $0.029 \%$. 进一步将面积担载量提高到 $6.0 \mathrm{mg} \cdot \mathrm{cm}^{-2}$, 电解液用量/ 硫正极质量 $\mathrm{E} / \mathrm{S}$ 比为 $20 \mu \mathrm{L} \cdot \mathrm{mg}^{-1}$ 时, $\mathrm{S} @$ Co-N $/ \mathrm{G}$ 的面积 比容量依然高达 $5.1 \mathrm{mAh} \cdot \mathrm{cm}^{-2}$. 同时增加电流密度, 在 $0.5 、 1 、 2$ 和 4 C 下, $\mathrm{S} @ \mathrm{Co}-\mathrm{N} / \mathrm{G}$ 可以分别释放出 942 、 $866 、 790$ 和 $618 \mathrm{mAh} \cdot \mathrm{g}^{-1}$ 比容量. 在 $1 \mathrm{C}$ 的大电流密度 下经过 500 次循环后, S@Co-N/G 正极依然可以保持 681 $\mathrm{mAh} \cdot \mathrm{g}^{-1}$ 的放电容量, 平均容量衰减率为 $0.053 \%$, 库仑 效率约为 $99.6 \%$, 表现出优异的倍率性能和循环稳定性.

利用金属单原子可以显著催化硫正极还原过程中 的动力学速率. 但是, 放电终态产物硫化锂为电子绝缘 体, 在充电初始容易针化团聚, 会严重降低锂硫电池的 库伦效率. 加快硫化锂的分解是实现硫化锂的活化氧化 的关键. Huang 等 ${ }^{[81]}$ 利用盐模板法制备了一种高金属钴 单原子含量 $(15.3 \%)$ 的钴/氮/碳单复合材料 $\left(\mathrm{Co}_{\mathrm{SA}}-\mathrm{N}-\mathrm{C}\right)$, 实现了对 Li-S 电池中(多)硫化锂转化与沉积的快速调 控. 理论和实验结果表明大量 Co- $\mathrm{N}_{4}$ 配位结构不仅能够 有效促进多硫化锂的吸附并催化加速其还原动力学, 而 且碳基体上大量分布的 CosA-N-C 位点能够诱导硫化锂 纳米颗粒的均匀沉积, 促进硫化锂快速分解. 当电流密 度 $>5 \mathrm{C}$, 电解液用量/硫正极质量 $\mathrm{E} / \mathrm{S}$ 比为 $12 \mu{\mathrm{L} \cdot \mathrm{mg}^{-1}}^{-1}$ 经过 1000 个周期循环后依然具有 $624 \mathrm{mAh} \cdot \mathrm{g}^{-1}$ 的可逆比 容量, 平均库伦效率高达 $99.5 \%$.

Niu 和 Song 等 ${ }^{[82]}$ 将镍单原子嵌入氮掺杂的石墨烯 中(Ni@NG), 通过表征发现 $\mathrm{Ni}$ 单原子与周围的 $\mathrm{N}$ 原子 形成 $\mathrm{Ni}-\mathrm{N}_{4}$ 的配位中心. 由于活泼的镍单原子的存在使 得复合体系具有良好的导电性, 而且 $\mathrm{Ni}^{-\mathrm{N}_{4}}$ 独特的结构 使得与多硫化锂(LiPS)间的结合能增大, 可以通过化学 吸附固定更多的多硫化锂, 并利用自身的催化活性可逆 地催化 LiPS 的转化, 加速充电和放电过程中的动力学 转化, 有效地限制了 LiPS 的溶解和穿梭效应(图 8). 因 此, 利用 $\mathrm{Ni} @ \mathrm{NG}$ 修饰后的隔膜, 在 $0.1 \mathrm{C}$ 电流密度下可 以释放出 $1387 \mathrm{mAh} \cdot \mathrm{g}^{-1}$, 对硫的初始有效利用接近 $86.8 \%$. 电解液用量/硫正极质量 $\mathrm{E} / \mathrm{S}$ 比为 $10.5 \mu \mathrm{L} \cdot \mathrm{mg}^{-1}$ 时, 可逆循环容量依然高达 $1301 \mathrm{mAh} \cdot \mathrm{g}^{-1}$. 即使将电流 密度增大至 $10 \mathrm{C}$ 时, 仍然保持 $612 \mathrm{mAh} \cdot \mathrm{g}^{-1}$ 的比容量, 每圈容量的平均衰减率为 $0.06 \%$. 硫正极的利用率得到 极大改善, 实用性得到极大提升.

\section{4 总结和展望}

功能化的碳基复合材料具有导电能力强、固硫作用 优异、催化效果显著的独特优势, 通过对碳基复合材料 形貌设计、体系优化和成分调控, 系统地研究金属碳基 材料不同成分、形貌和结构对电池性能的影响, 分析充 放电过程中硫及其中间相产物(多硫化锂)的电化学行

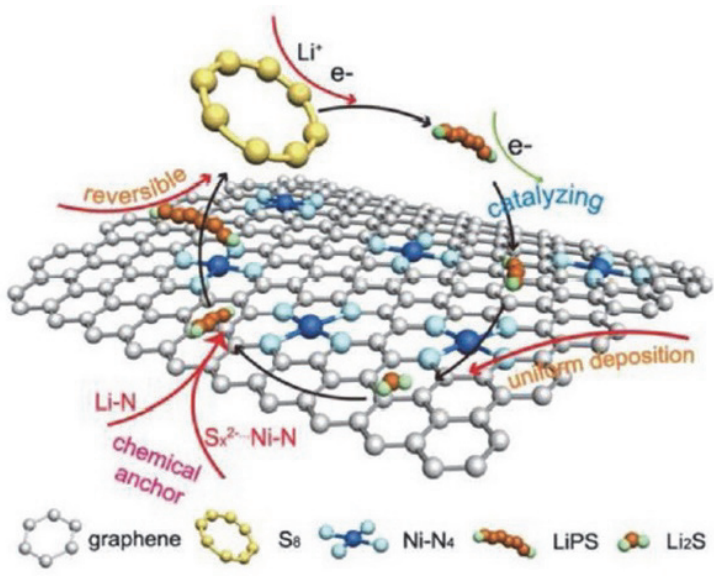

图 8 电化学过程中多硫化锂在 $\mathrm{Ni} @ \mathrm{NG}$ 表面的催化机理 ${ }^{[82]}$

Figure 8 The catalytic mechanism of the LiPS on the surface of $\mathrm{Ni@NG}$ in electrochemical process ${ }^{[82]}$

为, 揭示出电化学反应中载体材料与多硫化锂的相互作 用机理, 可以解决硫载量低、长循环中硫的利用率差、 以及大电流密度/高硫载量下固相多硫化锂转化不充分、 液相多硫化锂转化迟滞等问题, 为获得轻质化/多功能 的碳基复合储硫载体材料提供了可行策略. 但是, 该类 复合材料的实用化还存在许多挑战, 如熔融渗硫和传统 制浆的方法步骤繁琐、工艺复杂、装配技术要求高，不 利于工业化的便捷生产, 需要更多深入细致的研究以解 决这些问题．其次，碳基与金属基材料耦合得到的复合 载体的初始质量往往过大, 降低了复合硫正极的质量占 比, 削弱了复合硫正极的实际能量密度; 同时, 由于碳 基复合材料整体振实密度低导致体积能量密度过低. 协 调载体功能和质量的相关性是锂-硫电池实用化道路中 的关键因素. 最后, 无机硫源很难实现高硫含量 $(>$ $85 \%$ ), 而有机硫源虽然能够提高硫负载量, 但是其与现 有的碳基材料的耦合程度不佳，亟需开发与有机硫源相 匹配的碳基复合载体, 进一步满足实用化的需求.

\section{作者简介}

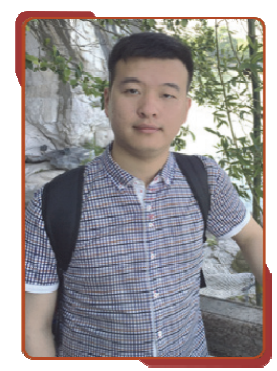

刘汉鼎, 目前在北京大学化学与分子工程学院李彦教授 课题组开展研究工作, 研究方向是碳纳米管及其衍生物的可 控制备及在储能领域的应用研究. 


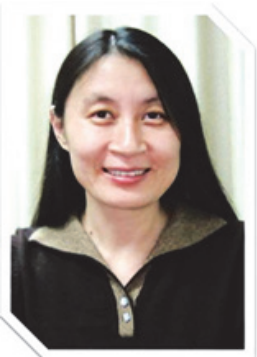

李彦, 北京大学化学与分子工程学院教授, 主要从事碳 纳米材料的研究, 在碳纳米管的可控生长、分离、表征和应用 等方面取得了一系列重要研究成果. 2011 年获得国家杰出青 年基金资助, 2013 年获聘教育部长江学者特聘教授, 以第一完 成人获 2017 年度教育部自然科学一等奖.

\section{References}

[1] Peng, Z.; Ding, H.; Chen, R.; Gao, C.; Wang, C. Acta Chim. Sinica 2019, 77, 681 (in Chinese). (彭正康, 丁慧敏, 陈如凡, 高超, 汪 成, 化学学报, 2019, 77, 681.)

[2] Wang, X.; Li, Y.; Du, L.; Gao, F.; Wu, Q.; Yang, L.; Chen, Q.; Wang, X.; Hu, Z. Acta Chim. Sinica 2018, 76, 627 (in Chinese). (王啸, 李 有须, 杜玲玉, 高福杰, 吴强, 杨立军, 陈强, 王喜章, 胡征, 化 学学报, 2018, 76, 627.)

[3] Liu, H.; Chen, Z.; Zhou, L.; Pei, K.; Xu, P.; Xin, L.; Zeng, Q.; Zhang, J.; Wu, R.; Fang, F. Adv. Energy Mater. 2019, 9, 1901667.

[4] Gao, L.; Bryan, B. A. Nature 2017, 544, 217.

[5] Liu, H.; Chen, Z.; Zhou, L.; Man, H.; Zeng, Q.; Pei, K.; Chen, G.; Wang, Q.; Song, Y.; Li, X. Energy Storage Mater. 2021, 41, 703.

[6] Ji, X.; Lee, K. T.; Nazar, L. F. Nat. Mater. 2009, 8, 500.

[7] Zhou, F.; Li, Z.; Luo, X.; Wu, T.; Jiang, B.; Lu, L. L.; Yao, H. B.; Antonietti, M.; Yu, S. H. Nano Lett. 2018, 18, 1035.

[8] Wang, Y.; Zhang, L.; Bi, J.; Yang, H.; Zhao, Z.; Mu, D.; Wu, B. Chin. J. Chem. 2020, 38, 1703.

[9] Chen, F.; Cheng, X.; Zhao, Z.; Wang, X. Acta Chim. Sinica 2021, 79,941 (in Chinese). (陈锋, 程晓琴, 赵振新, 王晓敏, 化学学报, 2021, 79, 941.)

[10] Liu, H.; Chen, Z.; Zhou, L.; Li, X.; Pei, K.; Zhang, J.; Song, Y.; Fang, F.; Che, R.; Sun, D. J. Mater. Chem. A 2019, 7, 7074.

[11] Liu, Z. F. Acta Phys.-Chim. Sin. 2020, 36, 2004003 (in Chinese). (刘 忠范, 物理化学学报, 2020, 36, 2004003.)

[12] Chen, K.; Sun, Z. H.; Fang, R. P.; Li, F.; Cheng, H. M. Acta Phys.-Chim. Sin. 2018, 34, 377.

[13] Sharma, P. K.; Cortes, M. A. L.; Hamilton, J. W.; Han, Y.; Byrne, J. A.; Nolan, M. Catal. Today 2019, 321, 9.

[14] Liu, H.; Chen, Z.; Man, H.; Yang, S.; Song, Y.; Fang, F.; Che, R.; Sun, D. J. Alloys Compd. 2020, 842, 155764.

[15] Chen, P.; Liu, Y.; Pan, P.; Fang, Y.; Shan, Q.; Feng, X.; Liu, R.; Lin, X.; Ma, Y. Chin. J. Inorg. Chem. 2021, 37, 1184 (in Chinese). (陈平, 刘逸然, 潘沛锋, 方亚男, 单淇, 冯晓苗, 刘瑞卿, 林秀婧, 马延 文, 无机化学学报, 2021, 37, 1184).

[16] Huang, Z.; Tang, M. L. J. Am. Chem. Soc. 2017, 139, 9412.

[17] Zhang, J.; Yang, C. P.; Yin, Y. X.; Wan, L. J.; Guo, Y. G. Adv. Mater. 2016, 28, 9539.

[18] Wujcik, K. H.; Pascal, T. A.; Pemmaraju, C.; Devaux, D.; Stolte, W. C.; Balsara, N. P.; Prendergast, D. Adv. Energy Mater. 2015, 5, 1500285.

[19] Xu, J.; Zhang, W.; Fan, H.; Cheng, F.; Su, D.; Wang, G. Nano Energy 2018, 51,73 .

[20] Yuan, Z.; Peng, H. J.; Hou, T. Z.; Huang, J. Q.; Chen, C. M.; Wang, D. W.; Cheng, X. B.; Wei, F.; Zhang, Q. Nano Lett. 2016, 16, 519.

[21] Lin, D.; Liu, Y.; Cui, Y. Nat. Nano 2017, 12, 194.

[22] Gao, J.; Lowe, M. A.; Kiya, Y.; Abruna, H. D. J. Phys. Chem. C 2011, 115, 25132.

[23] Yu, Z.; Zhang, J.; Liu, T.; Tang, B.; Yang, X.; Zhou, X.; Cui, G. Acta Chim. Sinica 2020, 78, 114 (in Chinese). (于喆, 张建军, 刘亭亭, 唐粼, 杨晓燕, 周新红, 崔光否, 化学学报, 2020, 78, 114.)

[24] Choi, J. W.; Kim, J. K.; Cheruvally, G.; Ahn, J. H.; Ahn, H. J.; Kim, K. W. Electrochim. Acta 2007, 52, 2075.

[25] Chang, D. R.; Lee, S. H.; Kim, S. W.; Kim, H. T. J. Power Sources
2002, $112,452$.

[26] Li, W.; Zhang, Q.; Zheng, G.; Seh, Z. W.; Yao, H.; Cui, Y. Nano Lett. 2013, 13, 5534 .

[27] Chen, J.-J.; Yuan, R.-M.; Feng, J.-M.; Zhang, Q.; Huang, J.-X.; Fu, G.; Zheng, M.-S.; Ren, B.; Dong, Q.-F. Chem. Mater. 2015, 27, 2048.

[28] Zhu, W.; Paolella, A.; Kim, C.-S.; Liu, D.; Feng, Z.; Gagnon, C.; Trottier, J.; Vijh, A.; Guerfi, A.; Mauger, A. Sustain. Energ. Fuels $2017,1,737$.

[29] Saqib, N.; Silva, C. J.; Maupin, C. M.; Porter, J. M. Appl. Spectrosc. 2017, 71, 1593.

[30] Dillard, C.; Singh, A.; Kalra, V. J. Phys. Chem. C 2018, 122, 18195.

[31] Barchasz, C.; Molton, F.; Duboc, C.; Leprêtre, J.-C.; Patoux, S.; Alloin, F. Anal. Chem. 2012, 84, 3973.

[32] Zou, Q.; Lu, Y.-C. J. Phys. Chem. Lett. 2016, 7, 1518.

[33] Zhang, L.; Wang, Y.; Niu, Z.; Chen, J. Carbon 2019, 141, 400.

[34] Yuan, L.; Yuan, H.; Qiu, X.; Chen, L.; Zhu, W. J. Power Sources 2009, 189,1141 .

[35] Dörfler, S.; Hagen, M.; Althues, H.; Tübke, J.; Kaskel, S.; Hoffmann, M. J. Chem. Commun. 2012, 48, 4097.

[36] Sun, L.; Li, M.; Jiang, Y.; Kong, W.; Jiang, K.; Wang, J.; Fan, S. Nano Lett. 2014, 14, 4044.

[37] Fu, A.; Wang, C.; Pei, F.; Cui, J.; Fang, X.; Zheng, N. Small 2019 , $15,1804786$.

[38] Zhou, G.; Xu, L.; Hu, G.; Mai, L.; Cui, Y. Chem. Rev. 2019, 119, 11042 .

[39] Kim, Y.; Han, H.; Noh, Y.; Bae, J.; Ham, M. H.; Kim, W. B. ChemSusChem 2019, 12, 824.

[40] Wei, Q.; Xiong, F.; Tan, S.; Huang, L.; Lan, E. H.; Dunn, B.; Mai, L. Adv. Mater. 2017, 29, 1602300.

[41] Hu, G.; Sun, Z.; Shi, C.; Fang, R.; Chen, J.; Hou, P.; Liu, C.; Cheng, H. M.; Li, F. Adv. Mater. 2017, 29, 1603835.

[42] Han, S. C.; Song, M. S.; Lee, H.; Kim, H. S.; Ahn, H. J.; Lee, J. Y. J. Electrochem. Soc. 2003, 150, A889.

[43] Zheng, G.; Yang, Y.; Cha, J. J.; Hong, S. S.; Cui, Y. Nano Lett. 2011, 11,4462 .

[44] Jian, Z.; Li, H.; Cao, R.; Zhou, H.; Xu, H.; Zhao, G.; Xing, Y.; Zhang, S. Electrochim. Acta 2019, 319, 359.

[45] Zhong, M.-E.; Guan, J.; Sun, J.; Guo, H.; Xiao, Z.; Zhou, N.; Gui, Q.; Gong, D. Electrochim. Acta 2019, 299, 600.

[46] Fang, R.; Li, G.; Zhao, S.; Yin, L.; Du, K.; Hou, P.; Wang, S.; Cheng, H.-M.; Liu, C.; Li, F. Nano Energy 2017, 42, 205.

[47] Yang, X.; Yan, N.; Zhou, W.; Zhang, H.; Li, X.; Zhang, H. J. Mater. Chem. A 2015, 3, 15314.

[48] Xiao, Z.; Xu, X.; Kong, D.; Liang, J.; Zhou, S.; Huang, X.; Yang, Q.; Zhi, L. J. Mater. Chem. A 2019, 7, 2009.

[49] Zhao, Y.; Wu, W.; Li, J.; Xu, Z.; Guan, L. Adv. Mater. 2014, 26, 5113.

[50] Xu, M.; Liang, T.; Shi, M.; Chen, H. Chem. Rev. 2013, 1, 3766.

[51] Pei, F.; Lin, L.; Fu, A.; Mo, S.; Ou, D.; Fang, X.; Zheng, N. Joule 2018, 2, 323 .

[52] Shan, J.; Liu, Y.; Su, Y.; Liu, P.; Zhuang, X.; Wu, D.; Zhang, F.; Feng, X. J. Mater. Chem. A 2016, 4, 314.

[53] Ji, L.; Rao, M.; Zheng, H.; Zhang, L.; Li, Y.; Duan, W.; Guo, J.; Cairns, E. J.; Zhang, Y. J. Am. Chem. Soc. 2011, 133, 18522.

[54] Lin, H.; Yang, L.; Jiang, X.; Li, G.; Zhang, T.; Yao, Q.; Zheng, G. W.; Lee, J. Y. Energ. Environ. Sci. 2017, 10, 1476.

[55] Kaiser, M. R.; Ma, Z.; Wang, X.; Han, F.; Gao, T.; Fan, X.; Wang, J.-Z.; Liu, H. K.; Dou, S.; Wang, C. ACS Nano 2017, 11, 9048.

[56] Song, J.; Gordin, M. L.; Xu, T.; Chen, S.; Yu, Z.; Sohn, H.; Lu, J.; Ren, Y.; Duan, Y.; Wang, D. Angew. Chem. Int. Ed. 2015, 127, 4399.

[57] Li, T.; Zhang, Y.; Chen, J.; Min, Y.; Wang, J. J. Inorg. Mater. DOI: 10.15541/jim20210303 (in Chinese). (李婷婷, 张阳, 陈加航, 闵宇 霖, 王久林, 无机材料学报, DOI: $10.15541 / \mathrm{jim} 20210303$ )

[58] Li, Z.; Zhang, J.; Lou, X. W. Angew. Chem. Int. Ed. 2015, 127, 12886 .

[59] Yu, M.; Ma, J.; Song, H.; Wang, A.; Tian, F.; Wang, Y.; Qiu, H.; Wang, R. Energ. Environ. Sci. 2016, 9, 1495.

[60] Li, R.; Zhou, X.; Shen, H.; Yang, M.; Li, C. ACS Nano 2019, 13, 10049 .

[61] He, J.; Luo, L.; Chen, Y.; Manthiram, A. Adv. Mater. 2017, 29, 1702707.

[62] Zhang, N.; Wei, Z.; Feng, T.; Wu, F.; Zhao, T.; Chen, R. Acta Phys. -Chim. Sin. 2022, 38, 2107009 (in Chinese). (张楠祥, 魏壮壮, 冯 涛, 吴锋, 赵腾, 陈人杰, 物理化学学报, 2022, 38, 2107009.) 
[63] Chen, K.; Sun, Z.; Fang, R.; Li, F.; Cheng, H. Acta Phys.-Chim. Sin. 2018, 34, 377 (in Chinese). (陈克, 孙振华, 方若翩, 李峰, 成会 明, 物理化学学报, 2018, 34, 377.)

[64] Zhang, M.; Chen, B.; Wu, M. Acta Phys.-Chim. Sin. 2022, 38, 2101001 (in Chinese). (张梦迪, 陈蓓, 吴明铂, 物理化学学报, 2022, 38, 2101001.)

[65] Zhang, J.; Li, Z.; Chen, Y.; Gao, S.; Lou, X. W. Angew. Chem. Int Ed. 2018, 130, 11110.

[66] Jiang, H.; Liu, X. C.; Wu, Y.; Shu, Y.; Gong, X.; Ke, F. S.; Deng, H. Angew. Chem. Int. Ed. 2018, 130, 3980.

[67] Hu, L.; Dai, C.; Liu, H.; Li, Y.; Shen, B.; Chen, Y.; Bao, S. J.; Xu, M. Adv. Energy Mater. 2018, 8, 1800709

[68] Li, W.; Ma, Q.; Zheng, Z.; Zhang, Y. Acta Chim. Sinica 2017, 75, 225 (in Chinese). (李宛飞, 马倩, 郑召召, 张跃钢, 化学学报, 2017, 75, 225.

[69] Habib, A.; Florio, F.; Sundararaman, R. J. Opt. 2018, 20, 064001.

[70] Huang, X.; Tang, J.; Luo, B.; Knibbe, R.; Lin, T.; Hu, H.; Rana, M.; Hu, Y.; Zhu, X.; Gu, Q. Adv. Energy Mater. 2019, 9, 1901872.

[71] Coey, J.; Berkowitz, A.; Balcells, L.; Putris, F.; Parker, F. Appl. Phys. Lett. 1998, 72, 734

[72] Sun, Z.; Zhang, J.; Yin, L.; Hu, G.; Fang, R.; Cheng, H.-M.; Li, F. Nat. Commun. 2017, 8,1 .
[73] Zheng, C.; Niu, S.; Lv, W.; Zhou, G.; Li, J.; Fan, S.; Deng, Y.; Pan, Z.; Li, B.; Kang, F. Nano Energy 2017, 33, 306.

[74] Li, R.; Peng, H.; Wu, Q.; Zhou, X.; He, J.; Shen, H.; Yang, M.; Li, C. Angew. Chem. Int. Ed. 2020, 59, 12129.

[75] Wu, H.; Li, Y.; Ren, J.; Rao, D.; Zheng, Q.; Zhou, L.; Lin, D. Nano Energy 2019, 55, 82.

[76] Wang, Y.; Zhang, R.; Pang, Y.-C.; Chen, X.; Lang, J.; Xu, J.; Xiao, C.; Li, H.; Xi, K.; Ding, S. Energy Storage Mater. 2019, 16, 228.

[77] Zhou, G.; Zhao, S.; Wang, T.; Yang, S.-Z.; Johannessen, B.; Chen, H.; Liu, C.; Ye, Y.; Wu, Y.; Peng, Y. Nano Lett. 2019, $20,1252$.

[78] Li, Y.; Zhou, P.; Li, H.; Gao, T.; Zhou, L.; Zhang, Y.; Xiao, N.; Xia, Z.; Wang, L.; Zhang, Q. Small Meth. 2020, 4, 1900701.

[79] Wang, C.; Song, H.; Yu, C.; Ullah, Z.; Guan, Z.; Chu, R.; Zhang, Y.; Zhao, L.; Li, Q.; Liu, L. J. Mater. Chem. A 2020, 8, 3421.

[80] Du, Z.; Chen, X.; Hu, W.; Chuang, C.; Xie, S.; Hu, A.; Yan, W.; Kong, X.; Wu, X.; Ji, H. J. Am. Chem. Soc. 2019, 141, 3977.

[81] Li, Y.; Wu, J.; Zhang, B.; Wang, W.; Zhang, G.; Seh, Z. W.; Zhang, N.; Sun, J.; Huang, L.; Jiang, J. Energy Storage Mater. 2020, 30, 250 .

[82] Zhang, L.; Liu, D.; Muhammad, Z.; Wan, F.; Xie, W.; Wang, Y.; Song, L.; Niu, Z.; Chen, J. Adv. Mater. 2019, 31, 1903955.

(Cheng, B.) 